\title{
Measuring the angular profile of the reflection of xenon scintillation light
}

\author{
C.P. Silva*, J. Pinto da Cunha, V. Chepel, A. Pereira, V. Solovov, P. Mendes, \\ F. Neves, M.I. Lopes \\ Department of Physics, LIP-Coimbra, University of Coimbra, 3004-516 Coimbra, Portugal
}

Available online 21 May 2007

\begin{abstract}
A chamber designed for measuring the angular distribution of reflected ultraviolet vacuum light is described. We report first measurements of the reflection profile of xenon scintillation light by a polished copper surface and by polytetrafluoroethylene (PTFE). (C) 2007 Elsevier B.V. All rights reserved.
\end{abstract}

PACS: 78.20.Ci; 29.40.M

Keywords: Reflectance; VUV; PTFE; Xenon scintillation; Liquid xenon

\section{Introduction}

Liquid xenon detectors based on scintillation are being used (or developed) for several applications, in particular dark matter search, neutrino experiments or medical imaging [1]. The response of such detectors depends on both the properties of the liquid (absorption and Rayleigh scattering) and on the reflectance of the container surfaces. These data are of extreme importance for the optimisation of the scintillation detectors and particularly as an input for the Monte Carlo simulations. However, since the scintillation light is in vacuum ultraviolet (VUV) at $\lambda \simeq 175 \mathrm{~nm}$, the absorption in air is high, making the measurements difficult.

The aim of this work is to measure the angular distributions of the xenon scintillation light reflected by materials used in liquid xenon chambers, especially Polytetrafluoroethylene (PTFE) which is used in the inner walls of liquid containers [2]. PTFE is known as a high reflectance diffuser having a reflectance of $\simeq 99 \%$ for wavelengths in the range from 350 to $1800 \mathrm{~nm}$ [3]. Much less, however, is known for wavelengths $\lambda \simeq 175 \mathrm{~nm}$ concerning both the reflectance and the angular distribu-

\footnotetext{
${ }^{*}$ Corresponding author. Tel.: + 351967144140 .

E-mail address: claudio@lipc.fis.uc.pt (C.P. Silva).
}

tion of the reflected light. Consequently, the Monte Carlo simulations usually assume a priori that the surface is purely Lambertian.

\section{Set-up}

For these measurements we designed and built a vacuum chamber, $40 \times 120 \times 30 \mathrm{~cm}^{3}$, made of stainless steel (lateral walls) and aluminium (top and bottom). To reduce the random light background, the inner walls of the chamber and most of the pieces of equipment were covered with black paper of low reflectivity (Thorlabs BFP1). The layout of the chamber is shown in Fig. 1.

The source of VUV light is a small cylindrical proportional counter with internal diameter of $40 \mathrm{~mm}$ and anode $0.5 \mathrm{~mm}$ filled with Xenon gas at $1 \mathrm{bar}$. An ${ }^{241} \mathrm{Am}$ source is mounted on one base at a distance of $1.5 \mathrm{~cm}$ from the central electrode emitting $5.5 \mathrm{MeV} \alpha$-particles along the counter axis. Under an applied voltage of $1.3 \mathrm{kV}$ the electrons extracted from the $\alpha$-tracks produce pulses of VUV light in the vicinity of the anode due to secondary scintillation which escapes the counter through a quartz window. The spectrum of this light is similar to scintillation in liquid Xenon, both are peaked at $175 \mathrm{~nm}$ [4]. The charge signal taken from the central anode is used for triggering. 


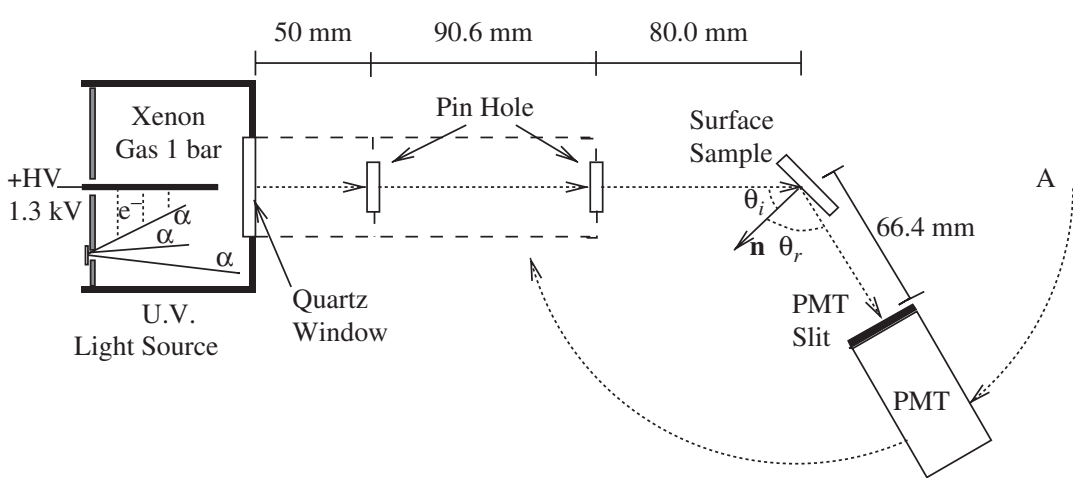

Fig. 1. Layout of the chamber.

In order to avoid the VUV light absorption (about $0.5 \mathrm{~cm}$ in air), the measurements are performed in an argon environment at 1 bar. The light is collimated with two pinholes, $2 \mathrm{~mm}$ in diameter, placed between the source and the sample to be studied. The sample is mounted in a structure that can rotate about the vertical axis allowing the angle of incidence to be changed accordingly. It can also be lifted up off the incident light beam (see Fig. 2). It is supported by three screws for fine tuning of the surface inclination. The structure has been carefully designed to avoid having any material behind the sample, as this might disturb the measurement of partially transparent materials (e.g. PTFE).

The VUV photons are detected by a photomultiplier (PMT) mounted on a moving arm that can rotate horizontally around the axis of the sample (see Fig. 2). The photomultiplier is a R1668 bi-alkaline photo-cathode tube from Hammamatsu with a quartz window. A slit of $2 \times 10 \mathrm{~mm}^{2}$ is placed in front of the PMT window (see Figs. 1 and 2). The measurements were carried out in photon counting mode.

The diagram of the electronics used in the experiments is represented in Fig. 3. The signal from the PMT is fed into a fast filter amplifier Ortec 579 (20 ns integration and $20 \mathrm{~ns}$ differentiation) and is discriminated, yielding a signal $150 \mathrm{~ns}$ long. The discrimination level was adjusted to detect single photo-electron signals.

The charge signal from the ionisation of the xenon is preamplified, differentiated by a $R C=3.3 \mu$ s and amplified by a fast amplifier. This signal once discriminated generates a gate $7.5 \mu$ s wide with average rate of about $1000 \mathrm{~Hz}$. This and the light signals are sent into a coincidence unit, which generates a digital output $150 \mathrm{~ns}$ wide. This unit generates as many outputs as input pulses within the gate length, as long as they are separated by more than $150 \mathrm{~ns}$. The delay between charge and light signal was observed to be less than $100 \mathrm{~ns}$ while the duration of the secondary light pulse is of the order of a microsecond. This delay has no effect in the measurements since the same fraction of light is lost in measuring both the incident and the reflected beam.

The output of the coincidence unit is read by a computer program through a data acquisition card from National Instruments. The number of coincidences per second is

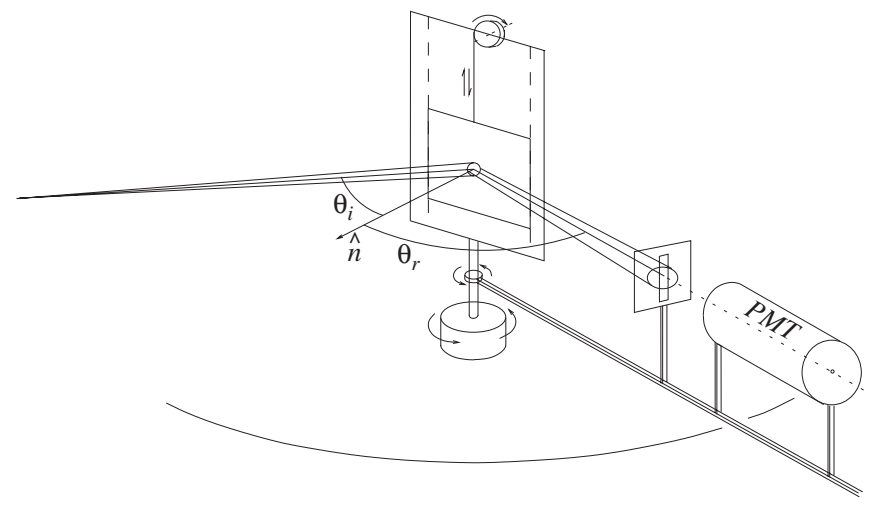

Fig. 2. Schematics of the positioning of the sample and PMT.

actually low enough for the number of detected photons being equal to the number of coincidences. In fact, the highest counting rate is obtained when the PMT is placed directly to the incident beam, in which case about 500 counts/s are measured.

The measurement sequences can be programmed in the computer, which also controls the motors that move the PMT and the sample.

The collimated light beam has an aperture of about $0.4^{\circ}$ and produces a spot on the sample of $3 \mathrm{~mm}$ in diameter. This translates into a spot disk of $4 \mathrm{~mm}$ at the PMT window. The beam profile has been scanned by moving the PMT with the $2 \mathrm{~mm}$ slit around position A with the sample removed (see Fig. 1) and found to agree with the above estimate.

The components of the system were aligned with a $\mathrm{He}-\mathrm{Ne}$ laser beam, namely the positions of pin holes, the PMT slit and the inclination of the samples relatively to the plane of reflection.

The measurements were performed in the plane of incidence but can be performed in other planes as well, as far as the sample is inclined relative to the rotation axis.

The incident angle, $\theta_{\mathrm{i}}$, and the reflection angle, $\theta_{\mathrm{r}}$, are both relative to the normal to the surface sample. They are calibrated using as reference the direction of the incident beam.

The reflectance is defined as the ratio between the intensities, the reflected over the incident. The intensity of 


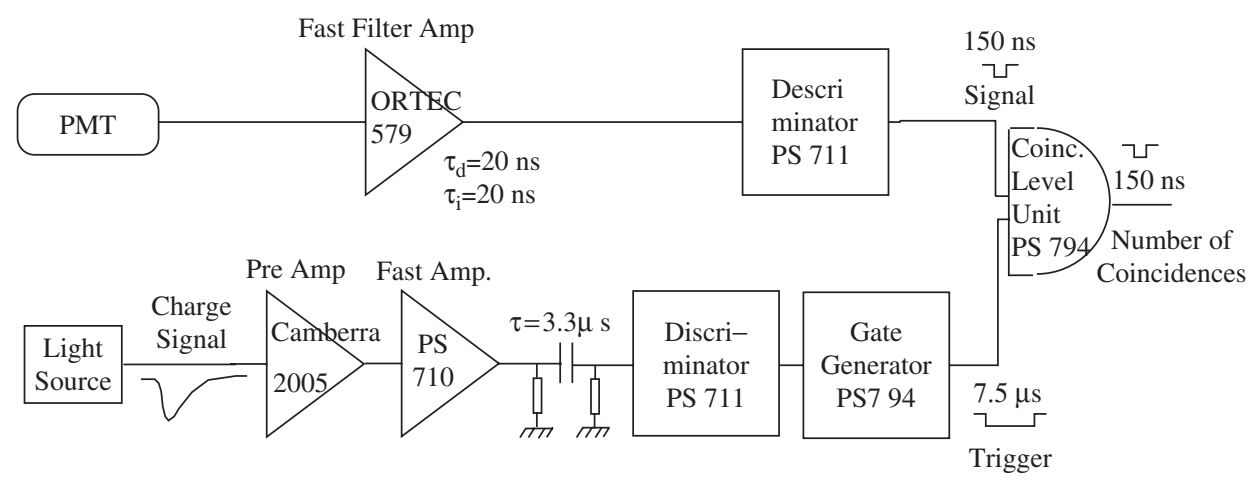

Fig. 3. Diagram of the electronics used in the experiment. PS stands for Phillips Scientific.

the incident beam is measured with the sample raised, letting the light go straight to the PMT. The PMT is moved to sample the entire beam spot. Adding up the photons for directions close to the incident beam direction we obtain a number that is proportional to the beam intensity, typically 1000 photons/s. With the sample up off the beam we also calibrate the PMT position carefully aligning the PMT with the incident beam. The position of the maximum marks the position where the PMT is perfectly parallel with the incident beam. The PMT can rotate $146^{\circ}$ relatively to the direction of the incident beam.

The position of the sample is calibrated putting the sample perfectly parallel to the incident beam. The sample can rotate $90^{\circ}$ relatively to the direction of incidence.

The background is measured for each $\theta_{\mathrm{i}}$ lifting the surface sample, the PMT is moved around as if it was measuring the reflectivity of the surface. These measurements show a background between 0.12 and 0.20 photons $/ \mathrm{s}$ along all the PMT course. This background comes principally from the PMT noise. Hence, the background is low enough to permit the observation of the Lambertian tails, which amount to about 0.6 photons/s for $\theta_{\mathrm{r}} \simeq 0^{\circ}$.

\section{Results}

Here we report preliminary results obtained for a sample of polished copper and a sample of PTFE. In order to validate the experimental procedure we measured the reflection of a surface of polished copper. In this case the reflection is mainly specular and can be studied easily as function of the angle of incidence. In this case the reflection and the incident angles are the same and the PMT is moved to sample the entire reflected beam spot. The ratio of intensities (reflected over incident light) is represented in Fig. 4. The data show a variation with the angle as expected from the Fresnel equations for absorbing media [5]. The values obtained are highly dependent of the oxidation of the surface. If the oxide layer is removed we observe an increase in reflectance as expected.

The fact that the measured angular dependence of the reflectance is fitted well with the Fresnel equations indicates

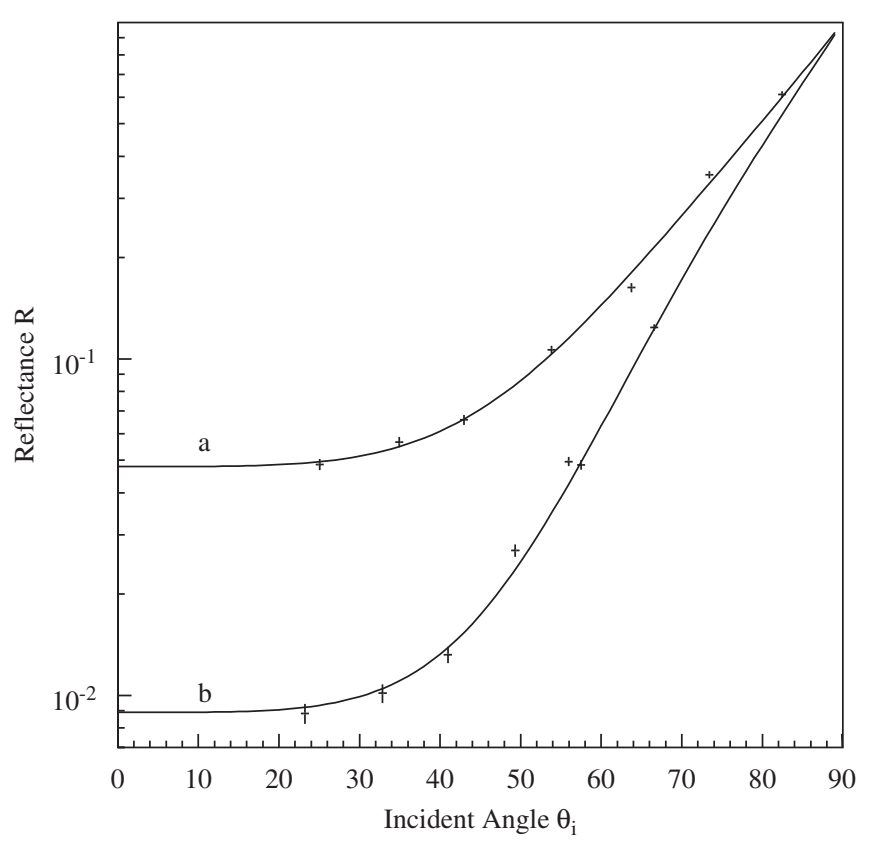

Fig. 4. The reflectance of a sample of copper as a function of the incident angle for two different levels of oxidation. The lines represent a fit to the data using the Fresnel equations for absorbing media [5] yielding to (a) $n=1.036 \pm 0.018$ and $\kappa=0.439 \pm 0.004$, and (b) $n=0.972 \pm 0.004$ and $\kappa=0.1925 \pm 0.003$, where $\tilde{n}=n+\mathrm{i} \kappa$ is the index of refraction for absorbing media.

good quality of the data and correctness of the experimental procedure.

We measured a sample of PTFE [6], the same material used to cover the walls of the liquid chamber used in Ref. [2]. The sample used is a $4 \times 3 \mathrm{~cm}^{2}$ sheet of PTFE, $1 \mathrm{~mm}$ thick, whose density is $1.09 \mathrm{~g} / \mathrm{cm}^{3}$. The intensity of the reflected beam at a given reflection angle, $\theta_{\mathrm{r}}$, is shown in Fig. 5 for $\theta_{\mathrm{i}}=30^{\circ}$. The polar plot in Fig. 5 shows the presence of both a specular lobe along the specular direction, and a distribution in $\cos \theta_{\mathrm{r}}$ characteristic of a Lambertian surface [7]. This measurement shows that this sample of PTFE is not purely diffuse for VUV light.

A narrow band filter for the xenon scintillation light $\lambda \simeq 175 \mathrm{~nm}$ and $\sigma_{\lambda}=8 \mathrm{~nm}$ with a peak transmittance of 


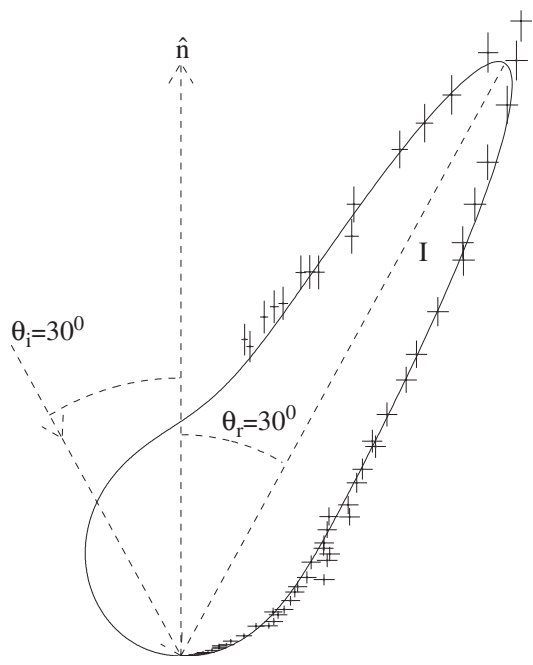

Fig. 5. Reflection profile of PTFE for VUV light in a polar representation, $I\left(\theta_{\mathrm{r}}\right)$, for incident light making $30^{\circ}$ with the surface normal (intensity is in arbitrary units). The specular lobe of reflection points towards the expected direction. The Lambert distribution is also seen. The solid line corresponds to a Lorentzian, describing the specular lobe, plus a cosine, describing the Lambertian component.

$12 \%$ was used in front of the PMT. The reflection profiles are the same with and without the filter and the ratio between them equals the transmittance of the filter, as expected. Hence, we are confident that no light other than xenon VUV is produced in the discharge.

\section{Conclusions}

A chamber for measuring the reflectance for VUV light has been constructed. The measurements done so far show that the experimental procedure is adequate to access the angular distributions of the reflected light. The observation of the reflectance by a polished surface shows the expected behaviour with the angle. The observed sample of PTFE shows the presence both specular and diffuse components for VUV light.

\section{Acknowledgements}

This work is supported by the FCT project POCTI/FP/ $63446 / 2005$, C. Silva was supported by the FCT fellowships SFRH/BD/19036/2004.

\section{References}

[1] M.I. Lopes, V. Chepel, IEEE Trans. Dielectr. Electr. Insul. 10 (2003) 994.

[2] V. Chepel, et al., Astroparticle Phys. 26 (1) (2006) 58.

[3] Weidner, et al., J. Opt. Soc. Am. 71 (7) (1981) (Appendix G).

[4] X. Schwentner, et al., Springer Tracks in Modern Physics, Springer, Berlin, 1985

[5] B. Michael, et al., Handbook of Optics, vol. II, McGraw-Hill, New York, 1995, p. 33.23.

[6] The sample used is referred to as ref. FP303050 in the GoodFellow's catalogue 2006.

[7] S.K. Nayar, et al., IEEE Trans. Pattern Anal. Mach. Intell. 13 (7) (1991) 611 